\title{
Quel Rôle pour les Acteurs Publics dans l'Incitation Privée aux Investissements ?*
}

\author{
Bruno Jullien ${ }^{\dagger}$ Jerome Pouyet ${ }^{\ddagger}$ Wilfried Sand-Zantman ${ }^{\S}$
}

September 13, 2017

\section{Introduction}

Les relations contractuelles entre, d'une part, entreprises privées et, d'autre part, autorités publiques structurent de nombreux pans de l'économie. C'est par exemple le cas lorsque des entreprises publiques construisent ou opèrent des services collectifs ou offrent des services privés subventionnés par les autorités publiques. Dans le domaine des transports, de l'énergie, des télécommunications ou de l'eau, il est courant que des collectivités locales fassent appel à des prestataires privés, au moins pour une partie de ces activités. Ces collectivités cherchent à optimiser la valeur de ces services pour les contribuables. C'est une des raisons invoquées pour faire appel à des prestataires privés. Mais ces activités peuvent également être prises en charge directement par des autorités publiques. L'objectif de cet article est donc de comprendre la nature des relations entre prestataires privés et autorités publiques, dans un cadre où les premiers doivent investir en amont et les seconds ont la capacité de fournir un service équivalent à celui proposé par les premiers.

Cet article a pour origine les problèmes de complémentarité ou de substituabilité entre acteurs publics et privés dans le secteurs des télécommunications. Depuis une

*Les auteurs remercient Orange pour son soutien intellectuel et financier. Ils remercient également les participants à la conférence organisée par la Revue Economique en l'honneur de David Martimort, ainsi qu'un rapporteur.

†Toulouse School of Economics-CNRS, Toulouse.

†THEMA-CNRS, ESSEC Business School, Université de Cergy-Pontoise. Adresse : ESSEC Business School, 3 avenue Bernard Hirsch, CS 50105 Cergy, 95021 Cergy-Pontoise Cedex, France.

$\S$ Toulouse School of Economics, Université de Toulouse Capitole et ESSEC Business School. 
dizaine d'années, ce secteur est marqué par une évolution technologique importante : l'introduction de la fibre. Le déploiement d'un réseau en fibre nécessite des investissements conséquents. Ces investissements sont encouragés par la régulation nationale et européenne ${ }^{1}$ et leur rentabilité est incertaine. Afin de rentabiliser ces investissements, les entreprises doivent proposer un prix reflétant le coût marginal de long terme et intégrant le risque de coût ou de demande pris en amont.

Certains acteurs publics (non régulés et pouvant se financer via l'imposition locale) peuvent être alors tentés d'entrer sur ce marché, une fois l'incertitude résolue. Une telle intervention par un acteur public local peut prendre plusieurs formes : soit une intervention directe en proposant des infrastructures publiques (ou subventionnées) que ce soit dans des zones où l'investissement privé est absent ${ }^{2}$ ou bien en parallèle aux infrastructures privées ; soit une intervention plus indirecte en faisant pression sur les acteurs privés pour qu'ils réduisent leur prix. ${ }^{3}$ Cette interaction peut avoir des conséquences non seulement ex-post (avant la résolution de l'incertitude concernant la rentabilité de l'investissement) mais également ex-ante (lorsque la rentabilité de l'investissement reste incertaine) sur les incitations à investir. Cette interaction a été notée dès 2009 par la Commission Européenne qui mentionne "it must be ensured that State aid does not crowd out market initiative in the broadband sector. [State intervention] could affect investment already made by broadband operators on market terms and significantly undermines the incentives of market operators to invest in the first place" 4 . Ainsi se posent à la fois la question de la bonne régulation sectorielle mais également celle du type de compétences qu'il est souhaitable d'accorder aux acteurs publics locaux. En d'autres termes, et pour résumer de façon quelque peu abrupte, doit-on contrôler a priori la nature des relations entre acteurs publics (collectivités locales bien souvent) et acteurs privés ? Dans l'affirmative, quelles règles doit-on imposer?

Pour aborder cette question, nous proposons un modèle simple dans lequel une

\footnotetext{
${ }^{1}$ Pour la France, le plan Très Haut Débit lancé en 2013 est évalué à 20 milliards d'euros, dont 3 milliards de subventions publiques.

${ }^{2}$ Par exemple, les "réseaux d'initiative publique" dans les zones peu denses où les collectivités locales financent, en partie sur fonds publics, la construction de réseaux loués ensuite aux opérateurs.

${ }^{3}$ Dans le cas de la fibre, il s'agit par exemple des prix de gros proposés aux acteurs industriels et commerciaux.

${ }^{4}$ Voir Public consultation on the Commission's Broadband Guidelines on the application of EU state aid rules to public funding of broadband networks, European Commission, 2009. Plus récemment, en France, voir les discussions entre les opérateurs et le gouvernement autour autour du "plan très haut débit" ("SFR fustige le plan très haut débit du gouvernement", Le Monde version électronique, daté du 19 juillet 2017).
} 
firme doit initialement choisir d'investir, ou pas, dans une technologie permettant de fournir un bien ex-post. Le coût lié à cet investissement est connu mais la demande est incertaine. Le prix que la firme sera susceptible de choisir est limité par les choix d'un régulateur. Cette régulation prend la forme d'un prix plafond pouvant être imposé aux clients et ce prix maximum est connu ex-ante au moment où la firme doit prendre sa décision d'investir. Sans tenir compte des autres acteurs, il s'agit simplement pour le régulateur de choisir un prix incitant à l'investissement privé.

Les choses deviennent plus complexes lorsque les actions des acteurs publics locaux sont prises en compte. Ces acteurs, dans les faits une collectivité locale avec à la fois des ressources propres et un objectif limité au surplus des consommateurs, peuvent intervenir une fois l'investissement privé réalisé et l'incertitude révélée. Cette intervention peut prendre deux modalités : une action directe sur le marché, par exemple par l'intermédiaire d'un investissement substitut à l'investissement privé et la mise en place d'une firme concurrente ; une négociation avec la firme privée, négociation se déroulant sous la menace de la mise en place de cette firme concurrente. Notre analyse est alors effectuée en deux temps.

Dans une première partie, nous considérons le cas où l'autorité publique locale peut prendre des actions consistant soit à investir pour concurrencer la firme privée, soit à négocier avec cette firme, après la réalisation et la révélation de l'incertitude sur la demande. Nous montrons qu'autoriser cette autorité à investir pour dupliquer les infrastructures privées sans permettre dans le même temps la négociation est néfaste pour la société. En effet, la possibilité pour la firme d'une concurrence dans certains états du monde la dissuade d'investir. Pour contrebalancer cet effet dépressif, le régulateur national doit d'augmenter le prix régulé de façon à ce que la firme puisse rentabiliser en moyenne son investissement. Mais cette hausse du prix et les distorsions qui s'ensuivent ne sont pas compensées par les bénéfices pour les consommateurs liés aux réductions de prix lorsque les autorités locales fournissent le bien. Si, maintenant, l'autorité locale est autorisée à négocier avec la firme privée sans pouvoir la concurrencer, elle est en mesure d'améliorer le surplus des consommateurs sans affecter les incitations privées. En effet, il n'y a pas de risque de hold-up expost dans ce cas ; le régulateur national peut alors inciter à l'investissement avec un prix régulé modéré, alors que la négociation permet d'utiliser un tarif binôme plutôt qu'un prix linéaire, supprimant ainsi les inefficacités. Enfin, on montre que la combinaison de la négociation et de la possibilité de concurrence publique ne permet pas d'augmenter le surplus social par rapport au cas précédent. En effet, même si la négociation permet d'avoir des tarifs plus flexibles, la possibilité de duplication réduit le pouvoir de négociation de la firme privée et donc ses incitations à investir. 
Pour contrebalancer cet effet, le régulateur national doit augmenter le prix régulé, qui structure le profit minimum conservé par la firme en cas de négociation. Mais l'existence d'un prix régulé suffisant pour inciter à l'investissement n'est alors plus garanti. Au final, on peut donc en conclure qu'accorder aux autorités la possibilité de dupliquer les investissements privés n'est pas socialement optimal, alors qu'autoriser des négociations entre autorités et firmes privées l'est.

La seconde partie de notre travail s'intéresse au cas où, au moment de la négociation, l'autorité locale n'est pas informée sur l'état de la demande alors que la firme est informée. Cette asymétrie a deux conséquences importantes : non seulement les autorités ne savent comment compenser la firme privée en cas de versement forfaitaire en contrepartie d'une baisse de prix mais ces autorités ne savent pas non plus si la duplication des infrastructures privées est socialement rentable. Cette seconde conséquence conduit à une réflexion sur la notion de crédibilité de la menace de duplication. Globalement, dans ce cadre d'information asymétrique, nous montrons que si les autorités publiques ne sont pas capables de concurrencer la firme privée, il est tout de même intéressant de les autoriser à discuter et négocier avec ces firmes. Les choses sont moins tranchées lorsque cette concurrence est possible, et la conclusion dépend de la force de la menace. Lorsque la menace est forte, il n'est plus possible d'inciter ex-ante les firmes privées à l'investissement, ce qui renchérit le coût total de fourniture du service. En revanche, lorsque cette menace existe, mais reste modérée, la possibilité de négocier entre acteurs publics locaux et firmes privées peut permettre à la fois d'inciter plus facilement à l'investissement d'un point de vue ex-ante et de diminuer les prix effectifs payés par les consommateurs ex-post.

Cet article puise ses sources dans différents types de littérature économique. A un niveau général, il fait écho aux articles relatifs à la régulation des monopoles (Laffont et Tirole, 1986, Lewis et Sappington, 1988, ou plus récemment Auriol et Picard, 2009). Mais notre problème a plusieurs spécificités. D'une part, il faut noter la présence de plusieurs entités tentant de contrôler l'activité de la firme privée, comme dans les contributions relatives aux multi-principaux (Martimort, 1996). Ensuite, les interactions entre production privée et production publique posent les problèmes de la limite de l'activité publique, comme dans la littérature liée aux Partenariats Public-Privé (Martimort et Sand-Zantman, 2006, Martimort et Pouyet, 2008 et Iossa et Martimort, 2015), ou bien simplement de coordination (Jullien, Pouyet et SandZantman, 2010). Enfin, le cadre de négociation entre la firme et les autorités locales est assez particulier car l'option de repli en cas d'échec de la négociation dépend d'éléments connus seulement de la firme, générant des contraintes de participation dépendantes du type (comme dans Jullien, 2000) mais également des croyances sur 
les actions hors équilibre (comme dans Cramton et Palfrey, 1995 ou Celik et Peters, 2011). Les difficultés liées à l'analyse de la négociation dans ce type de configuration ont été étudiées en détail dans Jullien, Pouyet et Sand-Zantman (2016), et nous utilisons leurs enseignements pour notre problème particulier.

L'article est construit de la façon suivante. Dans la Section 2, nous présentons le cadre général d'analyse. Dans la section 3, nous comparons différentes solutions réglementaires lorsque la négociation entre la firme et les autorités locales a lieu en information symétrique. Dans la section 4, nous élargissons l'analyse à des cas d'information asymétrique en discutant plus particulièrement la notion de menace de duplication et ses conséquences pour l'efficacité ex-ante et ex-post. La section 5 tire les enseignements de notre étude et conclut. Les preuves sont reléguées dans un Appendice.

\section{Le Modèle}

On considère une situation où un pays est composé d'un grand nombre de marchés locaux. Chaque marché est caractérisé par un paramètre $\theta$ mesurant la taille de sa demande. Pour chaque marché, ce paramètre est inconnu ex-ante et peut être modélisé comme une variable aléatoire de densité $f(\cdot)$ et de cumulative $F(\cdot)$ sur l'intervalle $[0, \bar{\theta}]$, en supposant également que le rapport $F(\theta) / f(\theta)$ est $_{\text {croissant }}{ }^{5}$. Ainsi, la demande dans chaque marché s'écrit $\theta D(p)$, avec $D(\cdot)$ une fonction décroissante du prix $p$ payé par le consommateur final, et on suppose que l'élasticité-prix de la demande $\varepsilon(p)=-D^{\prime}(p) p / D(p)$ est croissante ${ }^{6}$. De la même façon, le surplus du consommateur s'écrit $\theta W(p)$ avec $W(p)$ le surplus net du consommateur. Dans la mesure où chaque marché est ex-ante identique aux autres, nous nous concentrons sur l'analyse d'un seul marché.

La firme doit investir ex-ante pour pouvoir opérer sur le marché. Cet investissement représente un investissement matériel ou immatériel mais ne peut pas être récupéré en cas de sortie du marché. On note $k$ le montant de cet investissement réalisé par la firme avant que l'état de la demande soit connu. On suppose également (sans perte de généralité) que le coût marginal ex-post est égal à 0 .

\footnotetext{
${ }^{5}$ Cette condition de monotonicité du taux de hasard est satisfaite pour les grandes familles de distribution communément utilisées.

${ }^{6}$ Notons que cette hypothèse correspond à la deuxième loi marshallienne de la demande (voir Marshall, 1920).
} 
Le prix que peut choisir la firme est sujet à une régulation nationale déterminée ex-ante sous la forme d'un prix plafond. Ce prix est déterminé pour maximiser le surplus des consommateurs, ou, de façon équivalente, le surplus total sous contrainte d'équilibre budgétaire de la firme. Si ce prix plafond est choisi nationalement, il y a dans chaque marché une autorité locale dont l'objectif est la maximisation de l'utilité des résidents locaux. Cette autorité locale n'a pas de pouvoir de régulation à proprement parler. Elle a cependant la capacité soit de construire une infrastructure publique concurrente de l'infrastructure privée (on parle alors de duplication), soit de signer un contrat avec la firme privée pour baisser son prix unitaire en échange d'une compensation monétaire (on parle ici de négociation).

Dans le cas d'un investissement public, on suppose que le coût noté $K$ est supérieur au coût $k$ supporté par une firme privée. Cette hypothèse d'une plus grande efficacité privée n'est pas innocente, mais reflète l'idée que si les autorités publiques interviennent dans des secteurs où de grandes firmes privées sont présentes, il est raisonnable de penser qu'elles manqueront d'expertise. Notons également que si l'hypothèse inverse était retenue, il ne faudrait jamais encourager les firmes privées à investir et au contraire attendre la réalisation de la demande et éventuellement procéder ensuite à un investissement public. Supposer que $K \geq k$ est donc une hypothèse structurante mais nécessaire à la pertinence de notre analyse. Enfin, ce coût $K$ peut intégrer le coût des fonds publics en plus du simple coût technique, justifiant encore davantage notre hypothèse relative sur les coûts respectifs du privé et du public.

On peut s'intéresser à un premier cas de référence dans lequel les autorités locales n'interviennent pas. Il s'agit donc d'étudier une situation simple dans laquelle (1) le régulateur national choisit initialement le prix maximum unitaire final $\bar{p},(2)$ la firme décide d'investir ou pas dans ce marché, (3) le paramètre de demande $\theta$ est réalisé, (4) la firme choisit son prix.

En supposant que le prix de régulation $\bar{p}$ est inférieur au prix de monopole (défini comme le prix maximisant $p D(p)$ ), la firme va choisir le prix maximum autorisé $\bar{p}$ et donc obtenir sur ce marché un profit de $\theta \bar{p} D(\bar{p})$. A l'étape d'investissement, la firme ne connait pas la réalisation du paramètre de demande $\theta$. Elle va donc investir si et seulement si

$$
\mathbb{E}(\theta) \bar{p} D(\bar{p}) \geq k .
$$

On peut alors facilement déterminer le prix choisi à la première étape par le régulateur national. En effet, si son objectif est la maximisation du surplus du consommateur, 
comme ce surplus est décroissant avec le prix, il va choisir le prix minimum satisfaisant la contrainte (1). Si son objectif est le surplus total, défini par $S W=$ $\mathbb{E}(\theta) W(p)+\mathbb{E}(\theta) p D(p)$, il choisira le même prix. En effet $S W$ étant également décroissant avec le prix, le régulateur va encore choisir le prix minimum compatible avec un investissement de la firme.

Lemme 1 S'il existe un prix $\bar{p}$ tel que $\mathbb{E}(\theta) \bar{p} D(\bar{p})=k$, alors ce prix sera choisi par le régulateur national, que son objectif soit le surplus du consommateur ou bien le surplus total défini comme la somme du surplus du consommateur et du profit de la firme.

Dans ce premier cas de référence où il n'y a pas d'intervention de l'autorité locale, le niveau de bien-être obtenu est alors $\mathbb{E}(\theta) W(\bar{p})$. Dans les sections qui suivent, nous analysons les effets de l'intervention d'une autorité locale sur les incitations privées à l'investissement et sur les prix subis par les consommateurs.

\section{Duplication et Négociation : Information Symétrique}

Duplication. Nous analysons maintenant le rôle joué par l'autorité locale. Par rapport au cas de référence étudié dans la section précédente, nous introduisons une étape supplémentaire dans laquelle l'autorité locale peut décider de dupliquer l'investissement privé. Cette décision est prise ici après la réalisation de la demande, et en toute connaissance non seulement de celle-ci mais également du prix que pratiquerait la firme privée en l'absence de concurrence.

Pour juger de la pertinence d'un tel investissement pour l'autorité locale, il faut déterminer les conséquences sur les consommateurs/contribuables de ce marché. En cas d'investissement public, le prix optimal doit être fixé au coût marginal, ici égal à zéro. Notons que c'est aussi le prix qui résulterait d'une concurrence ex-post entre la structure publique et la firme privée en cas de duplication (c'est-à-dire en cas de double investissement par la firme privée et l'autorité locale). Le surplus des consommateurs/contribuables dans ce cas est alors donné par $\theta W(0)-K$. En l'absence d'investissement public, ce surplus est simplement $\theta W(p)$ où $p$ est le prix choisi par la firme privée. L'autorité locale va donc choisir de dupliquer l'investissement privé si et seulement si

$$
\theta W(0)-K \geq \theta W(p) \Leftrightarrow \theta \geq \hat{\theta}=\frac{K}{W(0)-W(p)} .
$$


Il y aura duplication lorsque la demande est assez élevée et le prix proposé par la firme s'éloigne suffisamment du coût marginal. Cette duplication a deux effets : un premier favorisant les consommateurs ex-post et un second défavorisant l'investissement exante. En effet, la duplication permet d'augmenter le surplus des consommateurs dans les cas où la demande est forte mais elle réduit les profits espérés de la firme et donc les incitations privées à investir. Pour induire un investissement privé, le régulateur national doit alors choisir un autre prix, noté $p^{D}$, tenant compte du comportement de l'autorité locale dans les cas où la demande est élevée. Formellement, ce nouveau prix de régulation permettant d'induire de l'investissement privé est tel que

$$
\int_{0}^{\hat{\theta}} \theta p^{D} D\left(p^{D}\right) d F(\theta)=k .
$$

Ce prix, s'il existe, est évidemment supérieur au prix optimal régulé sans possibilité de duplication. Du point de vue du bien-être social, il y a donc un arbitrage entre un surplus plus fort lorsque la demande est élevée et un surplus plus faible dans le cas contraire. Ce surplus s'écrit comme

$$
\int_{0}^{\hat{\theta}} \theta W\left(p^{D}\right) d F(\theta)+\int_{\hat{\theta}}^{\bar{\theta}}(\theta W(0)-K) d F(\theta) .
$$

On peut alors montrer le résultat suivant.

Proposition 1 En l'absence de négociation contractuelle entre la firme et les autorités locales, il est socialement optimal d'interdire la duplication des investissements privés par les autorités locales publiques.

Preuve. Voir l'Appendice 5.1.

Comme expliqué plus haut, la possibilité de duplication des investissements privés a des effets positifs pour les cas où la demande est forte et des effets négatifs lorsque la demande est faible. Par rapport au cas où l'autorité locale n'intervient pas, la consommation de service augmente lorsque la demande est élevée et diminue sinon. L'élasticité de la demande étant croissante avec le prix, les baisses de consommation qui résultent d'une hausse de prix dans certains états du monde ont un tel impact négatif sur les consommateurs qu'elles font plus que compenser les augmentations de consommation associées aux baisses de prix en cas de duplication.

Négociation. Une autre possibilité légale peut consister non pas à autoriser les autorités locales à dupliquer ex-post les investissements privés mais à investir lorsque 
qu'il n'y a pas d'investissement privé et à négocier avec les acteurs privés s'ils sont déjà présents sur le marché.

L'intérêt d'une telle négociation est d'obtenir des prix plus faibles pour les consommateurs, en échange d'un transfert compensatoire (une subvention) versé à la firme privée. En effet, la présence d'un coût fixe d'investissement conduit le prix régulé à être au-dessus du coût marginal, engendrant alors une inefficacité. Cette inefficacité peut être réduite en subventionnant la firme pour que celle-ci propose des prix plus proches, voire égaux, au coût marginal. Plus précisément, les autorités locales, pour maximiser le surplus des consommateurs locaux, ont intérêt à proposer un contrat à la firme privée dans lequel cette dernière propose un prix $p=0$ aux consommateurs en contrepartie d'une contribution indexée par la niveau de demande, contribution notée $T(\theta)$. Cette contribution doit être acceptée par la firme, et doit donc satisfaire la contrainte

$$
T(\theta)-k \geq \theta p D(p)-k \Leftrightarrow T(\theta) \geq \theta p D(p)
$$

Notons qu'il est toujours optimal pour l'autorité locale de faire une telle offre. En effet,

$$
\theta W(0)-\theta p D(p) \geq \theta W(p) \Leftrightarrow W(0) \geq W(p)+p D(p)
$$

puisque le surplus total (brut du coût fixe d'investissement) $W(p)+p D(p)$ est maximisé pour un prix égal au coût marginal (qui est nul ici).

On peut aussi s'intéresser à l'effet ex-ante d'un tel choix. Du côté de la firme, si $p$ est le prix régulé initialement choisi par le régulateur et que ce prix réussit à induire de l'investissement, on a toujours les bonnes incitations dans ce cadre avec négociation. Plus précisément

$$
\int_{0}^{\bar{\theta}} T(\theta) d F(\theta)-k \geq 0 \Leftrightarrow \mathbb{E}(\theta) p D(p) \geq k .
$$

Allons même plus loin, et supposons que le prix régulé soit juste égal au prix nécessaire pour induire de l'investissement, et donc que $p=\bar{p}$. Alors, le surplus ex-ante du consommateur (et le surplus social total) est donné par

$$
\begin{aligned}
\int_{0}^{\bar{\theta}}(\theta W(0)-T(\theta)) d F(\theta) & =\int_{0}^{\bar{\theta}}(\theta W(0)-\theta \bar{p} D(\bar{p})) d F(\theta) \\
& =\mathbb{E}(\theta) W(0)-k>\mathbb{E}(\theta) W(\bar{p})
\end{aligned}
$$


Ce cadre dans lequel l'autorité locale a la possibilité de négocier avec les acteurs, mais pas de dupliquer leur investissement ou de leur faire concurrence, permet d'obtenir un surplus espéré égal au surplus maximal réalisable dans l'économie. Et bien sûr, ce maximum est strictement supérieur pour les consommateurs (comme pour la société dans son ensemble, car le profit espéré de la firme est nul) au niveau obtenu lorsqu'il n'y a ni négociation, ni duplication. On peut alors énoncer la proposition suivante.

Proposition 2 En l'absence de possibilité de duplication, il est socialement optimal d'autoriser les autorités locales à négocier des aménagements tarifaires avec les firmes privées.

Preuve. Immédiate d'après le texte.

Négociation et duplication. On peut enfin s'intéresser aux cas où l'autorité locale peut à la fois dupliquer les investissements privés mais également contracter avec la firme ayant investi. Cette double possibilité ouvre la voie à des négociations d'un type différent. En effet, dans le cadre de négociation sans duplication, l'échec de cette négociation conduisait les différents acteurs en mettre en œuvre la solution simple d'un monopole réglementé. Maintenant, l'échec de la négociation peut conduire l'autorité locale à investir dans sa propre infrastructure. Et la menace d'un tel comportement a évidemment des conséquences sur l'issue de la négociation initiale. Pour que l'éventualité d'une duplication ait une influence sur les négociations, il faut que la menace d'un tel comportement soit crédible. Formellement, une menace de duplication des investissements privés sera crédible si et seulement si

$$
\theta W(0)-K \geq \theta W(p) \Leftrightarrow \theta \geq \hat{\theta}
$$

avec $\hat{\theta}$ défini dans l'équation (2). La possibilité de duplication ex-post change bien sûr les termes de la négociation.

Si $\theta<\hat{\theta}$, c'est-à-dire si la demande est faible, il est de connaissance commune qu'il n'est pas dans l'intérêt de l'autorité locale de dupliquer les infrastructures privées. Le contrat proposé à la firme privée sera donc le même que celui proposé en l'absence de possibilité de duplication.

Si au contraire $\theta \geq \hat{\theta}$, c'est-à-dire si la demande est élevée, il est dans l'intérêt de l'autorité locale de dupliquer l'infrastructure privée. Dans ce cas, la firme obtiendrait un profit nul. Elle est donc en position très défavorable et prête à accepter n'importe quel contrat lui garantissant au moins autant que ce minimum. L'autorité locale peut alors contraindre la firme privée à proposer un prix égal au coût marginal, ici 
nul, sans avoir besoin de faire de transfert ${ }^{7}$. Quel que soit le niveau de la demande, le prix pratiqué par la firme ex-post sera nul. Cependant, la firme sera compensée dans le premier cas, alors qu'elle ne le sera pas dans le second.

Dans cette configuration, la firme privée ne consentira à investir dans l'infrastructure que si ses profits dans les états du monde sans duplication sont suffisants pour compenser les coûts d'investissement. Formellement, il faut donc que

$$
\int_{0}^{\hat{\theta}} \theta p D(p) d F(\theta) \geq k .
$$

Le prix régulé doit donc être le même que celui obtenu dans le cas avec duplication, c'est-à-dire égal à $p^{D}$ défini dans l'équation (3). La différence est maintenant que si la duplication n'est pas crédible, l'autorité locale a la capacité de proposer un contrat à la firme qui, tout en laissant son profit inchangé, augmente le surplus des consommateurs. On peut calculer le bien-être espéré dans ce cas

$$
\int_{0}^{\hat{\theta}}\left(\theta W(0)-\theta p^{D} D\left(p^{D}\right)\right) d F(\theta)+\int_{\hat{\theta}}^{\bar{\theta}} \theta W(0) d F(\theta)=\mathbb{E}(\theta) W(0)-k .
$$

On voit qu'ici encore, le bien-être total est par construction égal au surplus espéré des consommateurs et correspond au surplus maximum atteignable. Cela peut paraître surprenant car le prix régulé $p^{D}$ doit être élevé, et donc très éloigné du coût marginal, pour garantir un investissement par la firme. Mais ce prix n'est jamais mis en œuvre en pratique, son rôle étant fondamentalement de garantir un point de statu quo assez élevé à la firme en cas de négociation pour l'inciter à investir ex-ante.

Ceci dit, le risque de duplication rend plus restrictives les conditions d'existence d'un prix incitant à l'investissement. Et comme rajouter la possibilité de duplication à la négociation n'affecte pas le bien-être social, il est compliqué de défendre l'extension des attributions des autorités locales.

Corollaire 1 Lorsque les négociations ont lieu en information symétrique entre acteurs publics et privés, il est (faiblement) dominant de ne pas autoriser les acteurs publics à concurrencer les acteurs privés.

Preuve. Immédiate d'après le texte.

\footnotetext{
${ }^{7}$ Notons qu'avec un tel contrat, la firme privée est indifférente entre accepter et refuser mais cette indifférence pourrait être rompue en offrant une compensation minime à la firme.
} 
Conclusions. Notre analyse montre qu'il est pertinent d'autoriser les autorités locales à intervenir sur les marchés. Mais cette intervention doit être contrôlée, au sens où elle doit complémenter, et non pas concurrencer, les décisions des firmes privées lorsque celles-ci sont déjà régulées au niveau national. Ce point est important car, les autorités locales bénéficiant de ressources propres, elles disposent d'un avantage substantiel pour concurrencer les firmes privées. Il faut donc réserver l'intervention publique aux cas où l'initiative privée est absente ou pour améliorer les modalités des offres privées.

\section{Duplication et Négociation : Information Asymétrique}

Présentation. Dans la section précédente, nous avons supposé que les négociations entre autorités publiques et firmes privées se faisaient en information symétrique. Pourtant, il semble plus raisonnable de penser que les firmes ont un avantage informationnel sur les autorités publiques. En effet, elles sont habituées à opérer sur les marchés, et sont donc plus susceptibles d'avoir une idée précise sur la profitabilité de chaque marché. Il faut donc s'interroger sur les pouvoirs à laisser aux autorités publiques dans ce cadre.

Commençons par analyser informellement les nouveaux enjeux émergeant dans cette configuration informationnelle.

Considérons tout d'abord le cas où l'autorité locale peut simplement négocier avec la firme ayant investi, sans possibilité d'investir ou de menacer de le faire. Si seule la firme connait l'état de la demande, elle aura la tentation de surévaluer celle-ci afin de négocier un transfert important en contre-partie d'une baisse de prix.

Lorsque l'autorité locale peut également dupliquer les investissements privés, la question de l'opportunité de faire une telle duplication se pose. Mais cette opportunité dépend de l'état de la demande, un élément connu uniquement de la firme privée. On a donc une incertitude sur la crédibilité de la menace de duplication, et donc un impact double de l'information sur la demande, à la fois sur le profit minimal à laisser à la firme privée (son profit de réserve) mais également sur le pouvoir de négociation des autorités publiques.

\subsection{Négociation sans Duplication}

Nous reprenons le cadre exposé plus haut dans lequel la firme apprend l'état de la demande après avoir procédé à l'investissement. Parallèlement, l'autorité publique 
peut lui proposer un contrat de façon à diminuer le prix proposé aux consommateurs. Comme cette autorité ne connait pas le type de la demande, on peut utiliser le principe de révélation et s'intéresser aux mécanismes directs et révélateurs (voir Laffont et Martimort, 2002) de la forme $(p(\theta), T(\theta))_{\theta \in \Theta}$. Les mécanismes sont proposés en prenant comme donné le prix de régulation, noté $\bar{p}$. On note $\pi(\theta)$ le profit de la firme privée quand elle accepte le contrat proposé par l'autorité locale et que $\theta$ est le paramètre de demande. Le contrat doit satisfaire les contraintes de participation (CP) et des contraintes de compatibilité incitative (CI). On peut montrer facilement que ces contraintes s'écrivent pour tous les $\theta$ de la façon suivante

1. $(\mathrm{CP}): \pi(\theta)=\theta p(\theta) D(p(\theta))-T(\theta)>\pi^{R}(\theta)=\theta \bar{p} D(\bar{p})$,

2. (CI) : $\dot{\pi}(\theta)=p(\theta) D(p(\theta))$ et $\dot{p}(\theta) \geq 0$.

En l'absence de duplication, l'analyse est assez proche de celles faites par Lewis et Sappington (1988) et Auriol et Picard (2008). Notons tout d'abord que le contrat va toujours conduire à saturer la contrainte de participation pour au moins un des types $^{8}$.

On peut en fait montrer que le contrat optimal a deux formes distinctes. Pour $\theta \geq \theta^{*}$, l'autorité locale ne va pas proposer de contrat à la firme. On aura donc $\pi(\theta)=\pi^{R}(\theta)$ et $p(\theta)=\bar{p}$ pour tous les types $\theta \geq \theta^{*}$. Pour des niveaux de demande plus faibles $\left(\theta \leq \theta^{*}\right)$, l'autorité proposera une baisse du prix associée à un transfert permettant à la firme d'obtenir un profit plus élevé que son profit de réserve. Ce résultat est une conséquence des contraintes incitatives. En effet, puisque $\pi(\cdot)$ est convexe et que le prix $p(\cdot)$ doit être croissant mais inférieur à $\bar{p}$, il n'est pas possible d'avoir un profit dans le contrat d'abord égal au profit de réserve $\pi^{R}$ puis supérieur.

En négligeant la condition de compatibilité incitative du second ordre ${ }^{9}$ et en utilisant les techniques classiques, on peut montrer que le problème de l'autorité locale est de choisir le type limite $\theta^{*}$ et un contrat $(p(\cdot), \pi(\cdot))$ pour les types $\theta \leq \theta^{*}$

\footnotetext{
${ }^{8}$ Dans ce cas contraire, l'autorité locale pourrait diminuer le montant transféré à la firme sans affecter les incitations.

${ }^{9} \mathrm{On}$ montre dans l'Appendice que cette condition est une conséquence de la condition de taux de hasard monotone et de l'hypothèse sur l'élasticité de la demande.
} 
tels que

$$
\max _{\left\{(p(\cdot), \pi(\cdot))_{\left.\theta \leq \theta^{*}, \theta^{*}\right\}}\right.} \int_{0}^{\theta^{*}}(\theta W(p(\theta))+\theta p(\theta) D(p(\theta))-\pi(\theta)) f(\theta) d \theta+\int_{\theta^{*}}^{\bar{\theta}} \theta W(\bar{p}) f(\theta) d \theta
$$

sous les contraintes $\forall \theta \in\left[0, \theta^{*}\right]: \dot{\pi}(\theta)=p(\theta) D(p(\theta))$,

$$
\text { et } \pi\left(\theta^{*}\right)=\pi^{R}\left(\theta^{*}\right) \text {. }
$$

Le détermination formelle du contrat optimal est reléguée dans l'appendice et permet d'obtenir le lemme suivant.

Lemme 2 On suppose que l'autorité locale ne peut pas dupliquer les investissement privés et on définit le prix $p^{*}(\theta)$ comme la solution de l'équation $\varepsilon\left(p^{*}(\theta)\right)=$ $\left(1+\frac{\theta f(\theta)}{F(\theta)}\right)^{-1}$ ainsi que $\theta^{*} \equiv \min \left\{\bar{\theta},\left(p^{*}\right)^{-1}(\bar{p})\right\}$. Le contrat optimal proposé par l'autorité locale est alors tel que

- pour $\theta \in\left[0, \theta^{*}\right]$, le prix est égal à $p^{*}(\theta)$ et donc croissant avec $\theta$;

- pour $\left.\theta \in] \theta^{*}, \bar{\theta}\right]$, le prix est constant et égal à $\bar{p}$;

- le profit de la firme est tel que $\pi^{*}(\theta)-\pi^{R}(\theta)$ décrồt pour $\theta \in\left[0, \theta^{*}\right]$ et $\pi^{*}(\theta)=$ $\pi^{R}(\theta)$ pour $\theta \in\left[\theta^{*}, \bar{\theta}\right]$.

Preuve. Voir l'Appendice 5.2.

Pour comprendre la forme de ce contrat, il faut considérer les contraintes incitatives. La solution de premier rang consiste à proposer un prix nul (on rappelle que le coût marginal est nul) et compenser la firme à la hauteur de son utilité de réserve. Mais l'autorité locale souffre d'un déficit informationnel et n'est donc pas capable de bien apprécier l'utilité de réserve de chaque type de firme. Ces firmes vont donc être tentées de surévaluer celle-ci. Ce sont les firmes qui ont une demande faible qui sont susceptibles de mentir sur la demande, et ce sont donc ces firmes qu'il va falloir compenser au-delà de leur utilité de réserve. Pour éviter que ces firmes ne manipulent stratégiquement l'information sur le niveau de la demande, la seule façon est de diminuer la demande lorsqu'une firme prétend que le type est élevé, ce qui passe par une augmentation des prix.

Mais les prix ne peuvent pas excéder le prix régulé. On a donc deux zones distinctes. Soit le paramètre de demande est faible et le prix sera faible. Soit le paramètre de demande est élevé et la seule façon d'inciter l'ensemble des firmes à 
révéler honnêtement le niveau de la demande conduit à ne pas proposer de contrat, ou, de façon équivalente, à proposer un contrat qui ne modifie pas l'allocation obtenue en l'absence de contrat.

Deux remarques pour terminer cette première analyse. Tout d'abord, la négociation sans duplication étant meilleure à la fois pour les firmes et le consommateurs, il est toujours préférable de l'encourager (comme en information complète). Ensuite, le régulateur peut dans ce cas choisir un prix plafond plus faible que le niveau $\bar{p}$ tout en continuant à inciter suffisamment les firmes privées à investir.

\subsection{Négociation avec Duplication}

Lorsque l'autorité locale a le droit d'investir dans les infrastructures, elle peut utiliser cette capacité pour améliorer son pouvoir de marchandage dans la négociation. Mais pour que cela soit efficace, il faut qu'il soit effectivement dans l'intérêt de l'autorité de dupliquer les investissements privés.

On a vu plus haut que cela était le cas lorsque le paramètre de demande était au moins égal à $\hat{\theta}$. En présence d'information asymétrique, c'est paradoxalement la firme privée qui sait si une telle duplication est dans l'intérêt de l'autorité publique, et donc si la menace de duplication est crédible ou pas. Ce sont donc les actions de la firme privée qui peuvent indiquer à l'autorité locale si une duplication est envisageable.

Pour le comprendre informellement, supposons que l'autorité locale fasse une offre basse (à savoir donnant lieu à un profit faible) à la firme. Cette dernière va accepter ou refuser l'offre en fonction à la fois de ce qu'elle sait de la demande (la connaissance d'une demande élevée a tendance à diminuer la probabilité d'acceptation de l'offre) mais également de ce qu'elle anticipe comme réaction de l'autorité si elle refuse leur offre. Et la réaction des autorités, c'est-à-dire leur décision de dupliquer ou pas en cas de refus, dépend de leur croyance sur l'état de la demande si une firme refuse son offre. Dans Jullien, Pouyet et Sand-Zantman (2016), cette situation est étudiée en détail et l'ensemble des équilibres possibles de ce type de jeu est caractérisé. Nous nous focalisons donc ici sur les équilibres les plus pertinents pour notre analyse.

Equilibre avec expropriation. Ce premier équilibre permet de comprendre l'interaction entre les croyances de l'autorité locale sur le niveau de demande $\theta$ si la firme refuse l'offre faite, et le type d'offre effectivement proposé par cette autorité. Supposons que 
si une firme refuse le contrat proposé, l'autorité forme les croyances que la demande est élevée. Cela la conduirait à dupliquer l'infrastructure privée si

$$
\bar{\theta} W(0)-K \geq \bar{\theta} W(\bar{p}) \Leftrightarrow \bar{\theta} \geq \hat{\theta}
$$

Sous cette condition, et avec ces croyances, chaque firme anticipe qu'un refus de sa part conduira l'autorité à dupliquer son infrastructure, et donc réduira son profit à zéro. Les firmes, quelle que soit la demande, sont donc disposées à accepter tous les contrats qui lui garantiraient au moins un profit nul. En particulier, un contrat dans lequel le prix d'usage est égal au coût marginal (ici nul) et les transferts nuls (soit, un contrat $(p=0, T=0)$ ) est un contrat d'équilibre, accepté par la firme quel que soit l'état réel de la demande. Dans ce cadre, aucune duplication ne survient à l'équilibre, et les croyances hors équilibre en cas de refus du contrat (un niveau de demande $\bar{\theta}$ ) garantissent l'acceptation du contrat.

Avec un tel équilibre, la firme ne fait aucun profit ex-post. Anticipant une expropriation ex-post, elle ne sera pas incitée à investir ex-ante, ce qui conduira l'autorité locale à investir si et seulement si $\mathbb{E}(\theta) W(0)-K \geq 0$.

Equilibre avec profit. Nous allons plutôt nous concentrer sur un deuxième équilibre dans lequel la firme peut conserver un profit positif ex-post. Cet équilibre suppose que les croyances sur la demande $\theta$ suivant le rejet d'un contrat sont basées sur les types qui gagneraient plus en l'absence de contrat (et sans duplication) qu'avec le contrat.

A contrat donné et en l'absence de duplication, on peut définir deux sous-ensembles de types, un premier (avec des demandes faibles) composé de types acceptant le contrat et un autre (avec les demandes élevées) composé de types susceptibles de le refuser. Dans ce cadre, la duplication est crédible si l'espérance des types appartenant au deuxième sous-ensemble est supérieure au niveau limite $\hat{\theta}$. Nous allons voir comment, et à quel coût, l'autorité publique locale peut proposer un contrat qui permette effectivement de rendre la duplication crédible.

Comme dans le cadre précédent, un contrat correspond du point de vue de la firme à un profil de profit et de prix en fonction du niveau de la demande. Si l'autorité publique propose à chaque firme au moins le profit qu'elle pourrait obtenir en l'absence de contrat, on a vu dans la section précédente le meilleur contrat que cette autorité peut proposer. Si le contrat conduit certains types de firme à obtenir moins qu'en l'absence de contrat, la menace de duplication en cas de refus doit être 
crédible. Pour assurer la crédibilité de cette menace, il faut que seules les firmes faisant face à une demande élevée soient susceptibles de refuser le contrat. On doit donc garantir aux firmes ayant une demande faible un profit strictement supérieur à leur profit en l'absence de contrat (c'est-à-dire si le contrat est refusé et que ce refus n'induit pas de duplication).

Notons par $\theta^{r}$ le niveau de la demande tel que $\mathbb{E}\left(\theta \mid \theta \geq \theta^{r}\right)=\hat{\theta}$. Le contrat doit donc être tel que $\pi(\theta)>\pi^{R}(\theta)$ pour tous les types $\theta<\theta^{r}$. Puisque pour des raisons de compatibilité incitative le contrat optimal doit générer un profit croissant et convexe, cette condition peut être remplacée par la condition locale suivante $\pi\left(\theta^{r}\right) \geq \pi^{R}\left(\theta^{r}\right)$. Le contrat optimal est alors la solution du programme suivant

$$
\begin{gathered}
\max _{\{p(\cdot), \pi(\cdot)\}} \mathbb{E}(\theta W(p(\theta))+\theta p(\theta) D(p(\theta))-\pi(\theta)) \\
\text { sous les contraintes } \forall \theta, \dot{\pi}(\theta)=p(\theta) D(p(\theta)), p(\theta) \in[0, \bar{p}], \quad \dot{p}(\theta) \geq 0, \\
\forall \theta, \pi(\theta) \geq 0, \text { et } \pi\left(\theta^{r}\right) \geq \pi^{R}\left(\theta^{r}\right) .
\end{gathered}
$$

On peut alors montrer la proposition suivante.

Proposition 3 On suppose que $\mathbb{E}(\theta) \leq \hat{\theta}<\bar{\theta}$.

1. Si $\theta^{r} \leq \mathbb{E}(\theta)$ alors l'autorité publique propose un contrat unique tel que $p(\theta)=0$ et $\pi(\theta)=\theta^{r} \bar{p} D(\bar{p})$ pour tous les types.

2. Si $\theta^{r} \in\left[\mathbb{E}(\theta), \bar{\theta}\left[\right.\right.$ alors il existe $\theta^{* *}<\theta^{r}$ tel que

$$
\begin{aligned}
\left(1-\varepsilon\left(p\left(\theta^{* *}\right)\right)\right)\left(\int_{\theta^{* *}}^{\theta^{r}} F(\theta) d \theta-\int_{\theta^{r}}^{\bar{\theta}}(1-F(\theta)) d \theta\right) & =\varepsilon\left(p\left(\theta^{* *}\right)\right) \int_{\theta^{* *}}^{\bar{\theta}} \theta f(\theta) d \theta .
\end{aligned}
$$

Le contrat est tel que $p(\theta)=\min \left(p^{*}(\theta), p^{*}\left(\theta^{* *}\right)\right)$ où $p^{*}(\cdot)$ est défini dans le Lemme 2. Le profit des firmes est croissant, supérieur à $\pi^{R}(\theta)$ pour $\theta<\theta^{r}$ et inférieur ou égal sinon.

3. Si $\theta^{r} \geq \bar{\theta}$ alors le contrat optimal est celui obtenu dans le lemme 2 .

Preuve. Voir l'Appendice 5.3. 
Il y a donc trois cas possibles. Le troisième correspond à la situation dans laquelle même en cas de demande très élevée la duplication n'est pas une menace crédible pour l'autorité locale. Le meilleur contrat est alors celui obtenu dans la section précédente, avec des rentes élevées pour les types faibles en échange d'un prix d'usage proche du coût marginal.

Les premier et deuxième cas sont plus intéressants. On peut les analyser en regardant le profil des rentes laissées aux firmes en fonction du paramètre de demande $\theta$ connu d'elles seules. Ce profil est construit pour, à la fois, permettre de satisfaire la contrainte incitative (c'est-à-dire inciter à révéler l'état de la demande) mais également rendre la menace de duplication crédible. Pour cela, il faut que seuls les types élevés soient susceptibles de refuser le contrat. Plus précisément, le profil des rentes est tel qu'accepter le contrat est une stratégie dominante pour les types faibles, alors que pour les types élevés c'est la stratégie optimale parce qu'un refus révèlerait que leur type est élevé et engendrerait une duplication de la part de l'autorité locale.

Dans le premier cas, on peut obtenir un tel profil de rentes en conservant le prix optimal, c'est-dire nul, pour tous les types. Dans le deuxième cas, le profil de prix est plus complexe. Celui-ci est globalement croissant pour permettre de satisfaire la contrainte incitative mais avec, au départ, une partie strictement croissante et, ensuite, une partie constante. Ce profil de prix permet de procéder au meilleur arbitrage entre efficacité et réduction des rentes laissées aux firmes. Le graphique suivant présente le profil de rentes induit par le contrat optimal dans ce deuxième cas.

\subsection{Sur l'Optimalité du Régime Négociation avec Duplica- tion}

Il s'agit pour finir de comprendre les implications de ce régime avec duplication sur les incitations à investir. On va distinguer deux régimes, suivant que $\theta^{r}$ soit supérieur ou inférieur à $\mathbb{E}(\theta)$. Notons que $\theta^{r}$ est défini par $\mathbb{E}\left(\theta \mid \theta \geq \theta^{r}\right)=\frac{K}{W(0)-W(\bar{p})}$.

Soit $\bar{p}$ le niveau régulé tel que sans duplication ou négociation, il y ait investissement de la firme. Cela signifie que $\mathbb{E}(\theta) \bar{p} D(\bar{p})=k$. Pour $\bar{p}<\arg \max _{p} p D(p)$, on a donc une relation monotone entre $\bar{p}$ et $k$ avec $\bar{p}(k)$ une fonction croissante de $k$. Pour caractériser nos deux régimes, on peut intégrer cette fonction dans la définition de $\theta^{r}$. On obtient alors une limite entre les deux régimes définie par la condition

$$
\mathbb{E}(\theta \mid \theta \geq \mathbb{E}(\theta))=\frac{K}{W(0)-W(\bar{p}(k))} .
$$




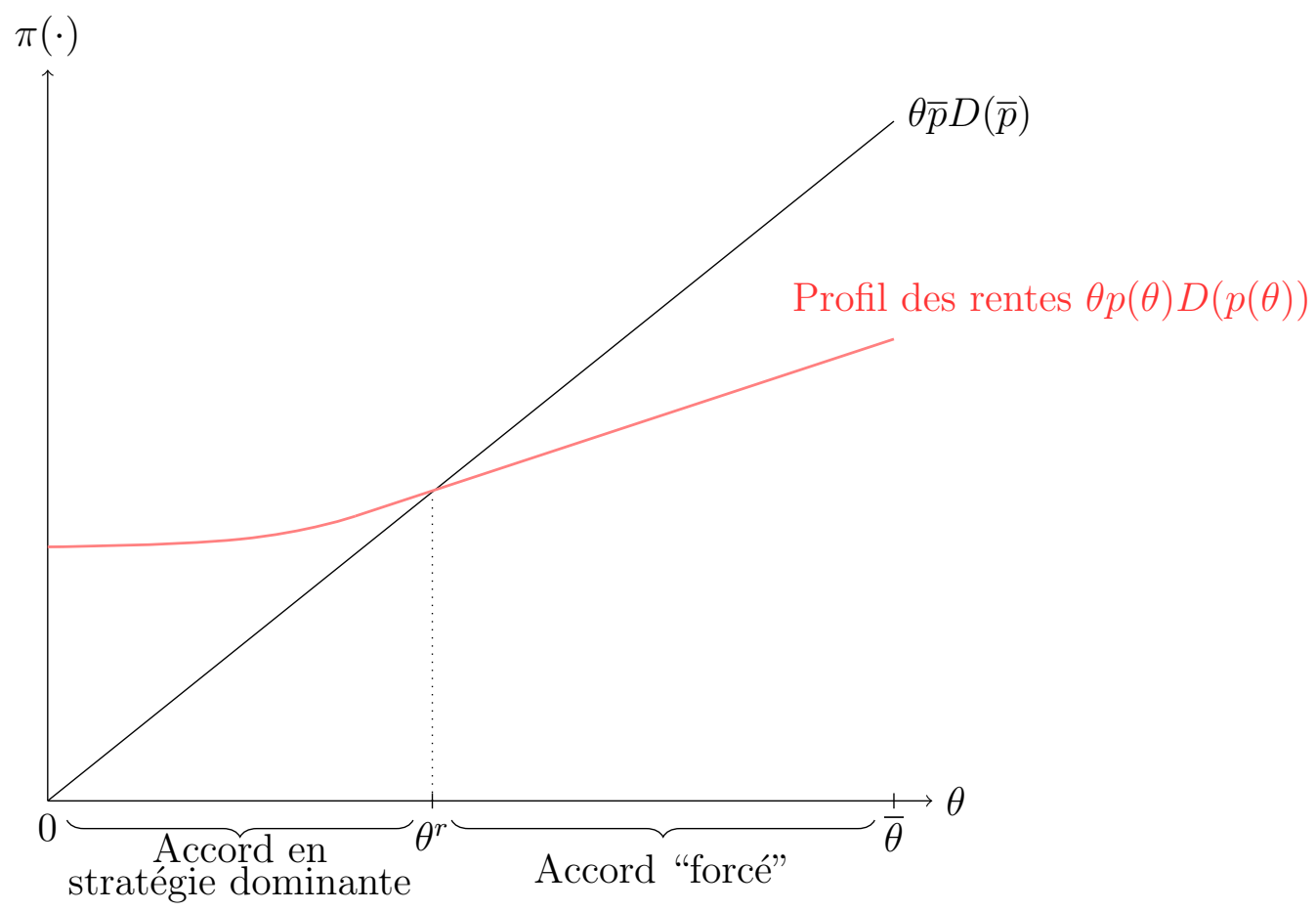

Regardons le premier régime dans lequel $\mathbb{E}(\theta \mid \theta \geq \mathbb{E}(\theta))>\frac{K}{W(0)-W(\bar{p}(k))}$, c'est-àdire $\theta^{r}<\mathbb{E}(\theta)$. Cela correspond à une situation dans laquelle $K$ et $k$ sont relativement proches, et donc la menace de duplication est forte. Le contrat optimal avec duplication est ici caractérisé par un prix nul et un paiement fixe donné à la firme égal à $\theta^{r} \bar{p} D(\bar{p})$. Par construction, ce paiement est inférieur à la somme nécessaire pour financer l'investissement. Ainsi, si le prix régulé correspond au cas sans duplication, l'autorisation de la duplication va dissuader l'investissement de la firme.

Est-il possible que modifier le prix $\bar{p}$ (à la hausse comme à la baisse) pour garantir un paiement espéré égal à $k$ ? On ne le sait pas, car augmenter $\bar{p}$ diminue $\hat{\theta}$ mais augmente $\bar{p} D(\bar{p})$. L'absence de monotonicité du profit par rapport au prix régulé rend donc difficile d'inciter à l'investissement en modifiant le prix. En résumé, dans ce premier cas, rien ne garantit qu'un régime avec menace de duplication soit préférable à un système sans négociation. En revanche, un système avec négociation mais sans duplication sera toujours meilleur.

On considère à présent le second régime dans lequel $\mathbb{E}(\theta \mid \theta \geq \mathbb{E}(\theta))<\frac{K}{W(0)-W(\bar{p}(k))}$ c'est-à-dire $\theta^{r}>\mathbb{E}(\theta)$. Cela correspond à la situation dans laquelle $K$ et $k$ sont rel- 
ativement éloignés, et donc la menace de duplication est faible. Le contrat optimal avec duplication est caractérisé par un profil de rentes croissant à partir d'une pente nulle et qui augmente jusqu'au point $\theta^{* *}<\theta^{r}$. A ce point, la pente de la fonction de profit est constante et égale à $\alpha=p^{*}\left(\theta^{* *}\right) D\left(p^{*}\left(\theta^{* *}\right)\right)<\bar{p} D(\bar{p})$ où $\bar{p}$ est le prix régulé. Le profit $\pi(\theta)$ est donc bornée inférieurement par la droite passant par $\theta^{r}$ et de pente $\alpha$, droite d'équation $\underline{\pi}(\theta)=\theta \alpha+\theta^{r}(\bar{p} D(\bar{p})-\alpha)$. L'espérance de cette droite est donnée par $\mathbb{E}(\underline{\pi}(\theta))=\mathbb{E}(\theta) \alpha+\theta^{r}(\bar{p} D(\bar{p})-\alpha)$. On veut comparer ce profit minimum avec le profit $\mathbb{E}\left(\pi^{R}(\theta)\right)=\mathbb{E}(\theta) \bar{p} D(\bar{p})$ qui assure un investissement privé. Nous obtenons

$$
\begin{aligned}
\mathbb{E}(\underline{\pi}(\theta))>\mathbb{E}\left(\pi^{R}(\theta)\right) & \Leftrightarrow \mathbb{E}(\theta) \alpha+\theta^{r}(\bar{p} D(\bar{p})-\alpha)>\mathbb{E}(\theta) \bar{p} D(\bar{p}) \\
& \Leftrightarrow \theta^{r}(\bar{p} D(\bar{p})-\alpha)>\mathbb{E}(\theta)(\bar{p} D(\bar{p})-\alpha) \\
& \Leftrightarrow \theta^{r}>\mathbb{E}(\theta),
\end{aligned}
$$

ce qui est exactement la définition de ce second régime.

Donc le profit généré avec la menace de duplication et un prix de régulation $\bar{p}$ est suffisant pour motiver la firme à investir ex-ante. En plus, les prix effectifs sont partout inférieurs à $\bar{p}$. Cette solution domine à la fois le régime sans négociation et le régime avec négociation sans duplication car les prix sont plus faibles pour tous les types intermédiaires ou élevés (les types $\theta>\theta^{r}$ ) lorsque la menace de duplication est introduite.

Notre analyse permet de donc de conclure avec la proposition suivante.

\section{Proposition 4}

(1) Quand la menace de duplication est forte, le prix de régulation optimal sans duplication ne permet pas d'inciter la firme à investir ex-ante et rien ne garantit qu'un prix alternatif permette de générer de l'investissement privé.

(2) Quand la menace de duplication est faible, autoriser la duplication augmente le profit de la firme et permet de diminuer les prix effectifs. Ce régime domine donc strictement le cas sans négociation mais aussi le cas avec négociation et sans duplication.

\section{Conclusion}

Cet article avait pour objet de s'interroger sur les moyens et les limites de l'action publique dans un cadre caractérisé par la présence de multiples autorités, nationales 
et locales. Il s'agissait en particulier de comprendre les conséquences sur l'investissement privé et le bien-être des consommateurs de l'imposition de contraintes ou au contraire de l'augmentation des moyens d'actions des autorités locales.

Une première conclusion robuste est qu'il est intéressant de favoriser les relations contractuelles entre ces autorités locales et les firmes qui fournissent des biens ou des services privés et soumis à une régulation nationale. Ces relations permettent d'obtenir une plus grande efficacité, par la plus grande flexibilité qu'elles permettent, tout en garantissant aux firmes le retour sur investissement anticipé au moment où cet investissement a été consenti.

Une seconde conclusion est qu'il peut être néfaste de laisser les autorités locales produire ces biens privés (soit directement, soit indirectement), car cette capacité modifie les modalités de négociations avec les firmes et met en danger la possibilité même d'un investissement privé. La force de cette menace dépend d'éléments très spécifiques (la structure d'information, les différentiels de coût entre public et privé, ...) mais oblige à penser les limites de la sphère publique. La flexibilité que permet la contractualisation entre autorités publiques et firmes privées doit donc aller de pair avec la préservation des incitations à l'investissement privé.

\section{Appendix}

\subsection{Preuve de la Proposition 1}

Nous nous intéressons à la différence de bien-être social en l'absence et avec duplication, soit

$$
A(k)=(\mathbb{E}(\theta) W(\bar{p}))-\left(\int_{0}^{\hat{\theta}} \theta W\left(p^{D}\right) d F(\theta)+\int_{\hat{\theta}}^{\bar{\theta}}(\theta W(0)-K) d F(\theta)\right),
$$

où $\bar{p}$ est tel que $\mathbb{E}(\theta) \bar{p} D(\bar{p})=k, \hat{\theta}$ est tel que $\hat{\theta}=K /\left(W(0)-W\left(p^{D}\right)\right)$ et $p^{D}$ est tel que $\int_{0}^{\hat{\theta}} \theta p^{D} D\left(p^{D}\right) d F(\theta)=k$.

Lorsque $k=0, \bar{p}=0$ et, de plus, $p^{D}=0$ et $\hat{\theta}=\bar{\theta}$. Les deux régimes sont alors équivalents et $A(0)=0$. 
Nous obtenons alors

$$
\begin{aligned}
A^{\prime}(k) & =-\mathbb{E}(\theta) D(\bar{p}) \frac{d \bar{p}}{d k}-\frac{d \hat{\theta}}{d k} f(\hat{\theta})\left(\hat{\theta} W\left(p^{D}\right)-\hat{\theta} W(0)+K\right)+\frac{d p^{D}}{d k} \int_{0}^{\hat{\theta}} \theta D\left(p^{D}\right) d F(\theta) \\
& =-\mathbb{E}(\theta) D(\bar{p}) \frac{d \bar{p}}{d k}+\int_{0}^{\hat{\theta}} \theta d F(\theta) D\left(p^{D}\right) \frac{d p^{D}}{d k}
\end{aligned}
$$

où la deuxième égalité utilise la définition de $\hat{\theta}$. En utilisant les définitions de $\bar{p}, p^{D}$ et de $\hat{\theta}$, et en introduisant la notation $\pi(p)=p D(p)$, nous obtenons

$$
\begin{aligned}
\left(\frac{d \bar{p}}{d k}\right)^{-1} & =\mathbb{E}(\theta) \pi^{\prime}(\bar{p}), \\
\left(\frac{d p^{D}}{d k}\right)^{-1} & =\int_{0}^{\hat{\theta}} \theta d F(\theta) \pi^{\prime}\left(p^{D}\right)+\pi\left(p^{D}\right) \hat{\theta} f(\hat{\theta}) \frac{d \hat{\theta}}{d p^{D}} \\
& =\int_{0}^{\hat{\theta}} \theta d F(\theta) \pi^{\prime}\left(p^{D}\right)-\pi\left(p^{D}\right) \hat{\theta}^{2} f(\hat{\theta}) \frac{D\left(p^{D}\right)}{W(0)-W\left(p^{D}\right)}
\end{aligned}
$$

Remarquons que $\frac{d \bar{p}}{d k}>0$ car $\bar{p} \leq \arg \max _{p} \pi(p)$. De façon similaire, $p^{D}$ est défini comme la plus petite solution de l'équation $\left(1-F\left(\hat{\theta}\left(p^{D}\right)\right)\right) \pi\left(p^{D}\right)=k$. Pour $k=0$ la solution est égale à 0 . Par continuité, pour des valeurs de $k$ suffisamment faibles, une telle solution existe et un argument standard montre que cette solution croît avec $k$, ou bien $\frac{d p^{D}}{d k}>0$. Nous retiendrons donc cette condition par la suite.

Nous pouvons alors obtenir la réécriture suivante

$$
A^{\prime}(k)=\frac{-D(\bar{p})}{\pi^{\prime}(\bar{p})}+\frac{D\left(p^{D}\right)}{\pi^{\prime}\left(p^{D}\right)-B}
$$

où $B=\frac{\hat{\theta}^{2} f(\hat{\theta}) \pi\left(p^{D}\right) D\left(p^{D}\right)}{\int_{0}^{\hat{\theta}} \theta d F(\theta)\left(W(0)-W\left(p^{D}\right)\right)}>0$ et $\pi^{\prime}\left(p^{D}\right)-B>0$ puisque $\frac{d p^{D}}{d k}>0$, et $\pi^{\prime}(\bar{p}), \pi^{\prime}\left(p^{D}\right) \geq$ 0 puisque $\bar{p} \leq p^{D} \leq \arg \max _{p} \pi(p)$.

Notons alors que

$$
\frac{D\left(p^{D}\right)}{\pi^{\prime}\left(p^{D}\right)-B} \geq \frac{D\left(p^{D}\right)}{\pi^{\prime}\left(p^{D}\right)}
$$

et

$$
\frac{D\left(p^{D}\right)}{\pi^{\prime}\left(p^{D}\right)} \geq \frac{D(\bar{p})}{\pi^{\prime}(\bar{p})} \Leftrightarrow \frac{\pi^{\prime}(\bar{p})}{D(\bar{p})} \geq \frac{\pi^{\prime}\left(p^{D}\right)}{D\left(p^{D}\right)} \Leftrightarrow 1-\varepsilon(\bar{p}) \geq 1-\varepsilon\left(p^{D}\right),
$$

inégalité qui est toujours satisfaite puisque l'élasticité-prix de la demande est crois- 
sante par hypothèse. Cela implique donc que $A^{\prime}(k) \geq 0 \forall k \geq 0$, ce qui conclut la preuve.

\subsection{Preuve du Lemme 2}

La caractérisation du contrôle optimal est faite en deux étapes, tout d'abord en regardant le contrat sur l'intervalle $\left[\theta, \theta^{*}\right]$ et ensuite en optimisant par rapport à $\theta^{*}$.

La première partie est résolue en utilisant le principe de Pontryagin. On définit le Hamiltonien du problème, en notant $\mu$ la variable de co-état, comme

$$
H=(\theta W(p(\theta))+\theta p(\theta) D(p(\theta))-\pi(\theta)) f(\theta)+\mu(\theta) p(\theta) D(p(\theta)) .
$$

En utilisant les théorèmes pour les objectifs concaves exposés par Seierstad et Sydsaeter (1977), on obtient les conditions suffisantes suivantes

- $p(\cdot)$ doit maximiser $H$, soit $p(\theta) \in \arg \max _{p} \theta W(p)+\left(\theta+\frac{\mu(\theta)}{f(\theta)}\right) p D(p)$;

- $\dot{\mu}(\theta)=-\frac{\partial H}{\partial \pi}=f(\theta)$;

- $\dot{\pi}(\theta)=p(\theta) D(p(\theta))$;

- $\mu(0)=0$.

Les conditions précédentes impliquent que $\mu(\theta)=F(\theta)$ et donc que $p(\theta)=p^{*}(\theta)$ où $p^{*}(\theta)$ est défini implicitement par

$$
\theta p^{*}(\theta) D^{\prime}\left(p^{*}(\theta)\right)+\frac{F(\theta)}{f(\theta)}\left(D\left(p^{*}(\theta)\right)+p^{*}(\theta) D^{\prime}\left(p^{*}(\theta)\right)\right)=0 \Leftrightarrow \varepsilon\left(p^{*}(\theta)\right)=\frac{\frac{F(\theta)}{f(\theta)}}{\theta+\frac{F(\theta)}{f(\theta)}} .
$$

Puisque $\varepsilon(p)$ et $\frac{\frac{F(\theta)}{f(\theta)}}{\theta+\frac{F(\theta)}{f(\theta)}}$ sont croissants, $p^{*}(\theta)$ est également croissant.

La seconde partie de la preuve consiste à déterminer le type limite $\theta^{*}$. En se basant sur les résultats de contrôle optimal (voir Seierstad et Sydsaeter, 1987, chapitre 5, Théorème 17), ce niveau limite est donc solution de

$$
\max _{\theta^{*}} \int_{0}^{\theta^{*}}\left(\theta W\left(p^{*}(\theta)\right)+\theta p^{*}(\theta) D\left(p^{*}(\theta)\right)-\pi(\theta)\right) f(\theta) d \theta+\int_{\theta^{*}}^{\bar{\theta}} \theta W(\bar{p}) f(\theta) d \theta .
$$


La condition du premier ordre est

$$
\theta^{*} W\left(p^{*}\left(\theta^{*}\right)+\theta^{*} p^{*}\left(\theta^{*}\right) D\left(p^{*}\left(\theta^{*}\right)\right)-\pi\left(\theta^{*}\right)-\theta^{*} W(\bar{p})=0 .\right.
$$

Par continuité, on doit avoir $\pi\left(\theta^{*}\right)=\theta^{*} \bar{p} D(\bar{p})$, donc cette condition est réécrite comme

$$
\theta^{*}\left(W\left(p^{*}\left(\theta^{*}\right)+p^{*}\left(\theta^{*}\right) D\left(p^{*}\left(\theta^{*}\right)\right)-W(\bar{p})-\bar{p} D(\bar{p})\right)=0\right.
$$

Puisque $W(p)+p D(p)$ et $p^{*}(\theta)$ sont monotones, l'objectif est quasi-concave en $\theta^{*}$. On obtient alors $p^{*}\left(\theta^{*}\right)=\bar{p}$ pour la solution intérieure, c'est-à-dire $\theta^{*}=\left(p^{*}\right)^{-1}(\bar{p})$ si $\theta^{*}<\bar{\theta}$, et $\theta^{*}=\bar{\theta}$ sinon.

On remarque enfin que le prix $p^{*}(\theta)$ est continu et monotone croissant, ce qui garantit les conditions incitatives globales. De plus, le profit $\pi(\theta)$ est supérieur ou égal $\pi^{R}(\theta)$, ce qui garantit que les contraintes de participation sont satisfaites.

\subsection{Preuve de la Proposition 3}

On remarque tout d'abord que $\pi\left(\theta^{r}\right)=\pi^{R}\left(\theta^{r}\right)$ est la seule contrainte liante, les autres contraintes de participation et de crédibilité étant alors satisfaites. En utilisant les résultats des théorèmes 3 et 4 de Jullien (2000), la solution optimale est caractérisée par

- un profil de prix $p(\theta)$ continu et croissant ;

- une fonction de distribution cumulative $\gamma^{*}(\theta)$ telle que $\gamma^{*}(\theta)=0$ pour $\theta<\theta^{r}$ et $\gamma^{*}(\theta)=1$ pour $\theta \geq \theta^{r}$

- un type limite $\theta^{* *}$ tel que

- $p(\theta)=p^{*}(\theta)<\bar{p}$ si $\theta<\theta^{* *}, p(\theta)=p^{*}=p^{*}\left(\theta^{* *}\right) \leq \bar{p}$ si $\theta \geq \theta^{* *}$;

- si $p^{*}>0$, on doit avoir pour tout $\tau \in\left[\theta^{* *}, \bar{\theta}\right]$

$$
\left.\int_{\theta^{* *}}^{\tau} \frac{\partial}{\partial p}\left(\theta W(p)+\left(\theta+\frac{F(\theta)-\gamma^{*}(\theta)}{f(\theta)}\right) p D(p)\right)\right|_{p=p^{*}} f(\theta) d \theta \geq 0
$$

et si $p^{*}<\bar{p}$, on doit avoir pour tout $\tau \in\left[\theta^{* *}, \bar{\theta}\right]$

$$
\left.\int_{\tau}^{\bar{\theta}} \frac{\partial}{\partial p}\left(\theta W(p)+\left(\theta+\frac{F(\theta)-\gamma^{*}(\theta)}{f(\theta)}\right) p D(p)\right)\right|_{p=p^{*}} f(\theta) d \theta \leq 0
$$


On dérive l'expression d'intérêt dans notre problème, soit

$$
\begin{aligned}
\frac{\partial}{\partial p}\left(\theta W(p)+\left(\theta+\frac{F(\theta)-\gamma^{*}(\theta)}{f(\theta)}\right) p D(p)\right) & \\
& =\frac{F(\theta)-\gamma^{*}(\theta)}{f(\theta)} D(p)+\left(\theta+\frac{F(\theta)-\gamma^{*}(\theta)}{f(\theta)}\right) p D^{\prime}(p) \\
& =D(p)\left(\frac{F(\theta)-\gamma^{*}(\theta)}{f(\theta)}-\left(\theta+\frac{F(\theta)-\gamma^{*}(\theta)}{f(\theta)}\right) \varepsilon(p)\right) .
\end{aligned}
$$

Considérons le cas dans lequel $0<\theta^{* *}<\bar{\theta}$. La condition de continuité de la fonction de prix donne $p^{*}=p\left(\theta^{* *}\right)>0$. Alors

$$
\begin{aligned}
\frac{F(\theta)}{f(\theta)}-\left(\theta+\frac{F(\theta)}{f(\theta)}\right) \varepsilon\left(p^{*}\right) & >0 \text { pour } \theta>\theta^{* *} \operatorname{car} p^{*}<p^{*}(\theta) \\
\frac{F(\theta)-1}{f(\theta)}-\left(\theta+\frac{F(\theta)-1}{f(\theta)}\right) \varepsilon\left(p^{*}\right) & <0 \text { pour } \theta>\theta^{* *} \operatorname{car} \varepsilon\left(p^{*}\right)<1
\end{aligned}
$$

La partie gauche de $(A 1)$ est quasi-concave en $\tau$ alors que celle de $(A 2)$ est quasiconvexe. La condition (A1) est alors satisfaite pour tout $\tau$ si elle l'est pour $\tau=\bar{\theta}$, soit

$$
\int_{\theta^{* *}}^{\bar{\theta}}\left(\frac{F(\theta)-\gamma^{*}(\theta)}{f(\theta)}-\left(\theta+\frac{F(\theta)-\gamma^{*}(\theta)}{f(\theta)}\right) \varepsilon\left(p^{*}\right)\right) f(\theta) d \theta \geq 0 .
$$

Alors que la condition (A2) est satisfaite pour tout $\tau$ si

$$
\int_{\theta^{* *}}^{\bar{\theta}}\left(\frac{F(\theta)-\gamma^{*}(\theta)}{f(\theta)}-\left(\theta+\frac{F(\theta)-\gamma^{*}(\theta)}{f(\theta)}\right) \varepsilon\left(p^{*}\right)\right) f(\theta) d \theta \leq 0 .
$$

On obtient alors la condition (8), qui implique également que $\theta^{* *}<\theta^{r}$.

De la même façon, si $p^{*}=\bar{p}$, on obtient

$$
\int_{\theta^{* *}}^{\bar{\theta}}\left(\frac{F(\theta)-\gamma^{*}(\theta)}{f(\theta)}-\left(\theta+\frac{F(\theta)-\gamma^{*}(\theta)}{f(\theta)}\right) \varepsilon(\bar{p})\right) f(\theta) d \theta \geq 0 ;
$$

alors que pour $p^{*}=0$, on obtient

$$
\int_{0}^{\bar{\theta}}\left(\frac{F(\theta)-\gamma^{*}(\theta)}{f(\theta)}\right) f(\theta) d \theta=\theta^{r}-\mathbb{E}(\theta) \leq 0
$$

ce qui complète la preuve de la proposition. 


\section{Références}

Auriol E., et P. Picard (2009). "Government Outsourcing: Public Contracting with Private Monopoly", Economic Journal, Vol. 119, 14641493.

Cramton P., et T. Palfrey (1995). "Ratifiable Mechanisms: Learning from Disagreement", Games and Economic Behavior, Vol. 10 (2), 255-283.

Celik G., et M. Peters (2011). "Equilibrium Rejection of a Mechanism", Games and Economic Behavior, vol. 73 (2), 375-387.

Iossa E., et D. Martimort (2015) "The Simple Microeconomics of PublicPrivate Partnerships", Journal of Public Economic Theory, Vol. 17 (1), 4-48.

Jullien B. (2000). "Participation Constraints in Adverse Selection Models", Journal of Economic Theory, Vol. 93(1), 1-47.

Jullien B., Pouyet J. et W. Sand-Zantman (2010). "Public and Private Investments in Regulated Network Industries: Coordination and Competition Issues," Review of Network Economics, Vol. 9 (4), Article 3 .

Jullien B., Pouyet J. et W. Sand-Zantman (2016). "An Offer You Can't Refuse: Early Contracting with Endogenous Threat", The RAND Journal of Economics, Vol. 48 (3), 733-748.

Laffont J.J., et D. Martimort (2002). The Theory of Incentives: The Principal-Agent Model, Princeton University Press.

Laffont J.J., et J. Tirole (1986). "Using Cost Observation to Regulate Firms", Journal of Political Economy, Vol. 94 (3), 614-641.

Lewis T. et D. E. Sappington, (1988). "Regulating a Monopolist with Unknown Demand ", American Economic Review, Vol. 78(5), 986998.

Marshall A. (1920). Principles of Economics. Eighth ed., London:Macmillan and Co., Ltd.

Martimort D. (1992). "Multiprincipaux avec Anti-Sélection," Annales d'Economie et de Statistiques, 28, 1-38. 
Martimort D. et J. Pouyet (2008). "To build or not to build: Normative and Positive Theories of Public-Private Partnerships", International Journal of Industrial Organization, Vol. 26 (2), 393-411.

Martimort D. et W. Sand-Zantman (2006). "Signalling and the Design of Delegated Management Contracts for Public Utilities", The RAND Journal of Economics Vol. 37 (4), 763-782.

Seierstad A. et K. Sydsaeter, (1977). "Sufficient Conditions in Optimal Control Theory", International Economic Review, 18, 367-391. 